\title{
Analysis and Measures for Current Development of Popular Taekwondo in China
}

\author{
Tian Jin $\mathrm{He}^{1, \text { a * }}$ and $\mathrm{Li} \mathrm{Ma}$, b \\ ${ }^{1}$ Physical School of East China Jiaotong University, Nanchang, Jiangxi, China \\ 2Foreign Language School of East China Jiaotong University, Nanchang, Jiangxi, China \\ a57627550@qq.com, birisma@netease.com
}

Key Words: Popular Taekwondo; Current Situation of Development; problems, measures.

\begin{abstract}
With the fast development of Chinese society, sports have already become the necessary part in people's daily life. As a new sport item and fitness way, Taekwondo is widely loved by the numerous Chinese teenagers. Especially after winning the Olympic gold medals for several times after its short -term development, the Chinese public Taekwondo has been greatly promoted. By investigating mass related materials, this paper analyzes the reasons and existing problems in the promotion and development of Chinese popular Taekwondo, and also proposes some suggestions for its orderly and healthy development in the future.
\end{abstract}

\section{Introduction}

The source of Taekwondo can be traced back to the self-defense combatives in Korean Peninsula [1]. Basing on absorbing some technique skills of Chinese martial arts and Japanese karate, Taekwondo has formed its own technical system and unique style. Taekwondo has many good functions for people, such as keeping fitness, self-defense and self-cultivation, which exactly are in line with the trend of international mass sports [2]. But because it is a new sport item in China, there are really few relevant researches about it. By proposing some suggestions as well as analyzing the reasons and exiting problems of its current situation, this paper aims to promote the all-round and healthy development of Taekwondo in China.

\section{The Development of Popular Taekwondo in China}

The first launch of Taekwondo in China was in Taiwan. In 1966, there was Taekwondo class, and it became popular in 1970s. [3] As the founder of Taekwondo, General Choi Hong-Hee had attached great importance to the popularity of Taekwondo in China, and he thought this course had major significance. His visit to China with international Taekwondo delegation in 1986 was a huge success. The splendid performances in many Chinese cities earned many loyal fans who want to learn it.

Chinese Taekwondo Association was founded in 1995. And a year later, the First National Taekwondo Championships was held in Jinhua, Zhejiang province, about 33 teams and 308 athletes took part in it. 1n 1997, the founder of Shanghai Black Belt Taekwondo club, Wang Shuangzhong 
went to Shanghai alone and prompted Taekwondo in universities. With his assistance, there were 6 college Taekwondo clubs and many exchange competitions. After that, Shanghai Black Belt Taekwondo club was founded in 1999, and now it becomes the most influential Taekwondo club in China [4].

\section{The Main Reasons of the Fast Development of Popular Taekwondo in China}

Taekwondo is a sport advocating technique, etiquette, ethics and self-cultivation. Despite its short history in China, Taekwondo is fashionable rapidly in recent years, whose white sportswear, black belt and elegant figure have attracted numerous people, especially teenagers.

The Achievements of Athletics Taekwondo in China. Initially, the development of popular Taekwondo was stimulated by athletics Taekwondo. During the last 20 years, Chinese athletes have won a lot of champions in all kinds of international competitions [5]. Especially in the last four Olympic Games, there were 5 Chinese athletes won 7 gold medals. With the outstanding performance of Chinese Taekwondo athletes in international competitions, popular Taekwondo has entering into a period of rapid development. Lots of colleges and universities have many Taekwondo activities, higher sports institutions have set up Taekwondo major and numerous Taekwondo clubs have sprung up, over 500 clubs only in Beijin and Xi'an.

Emphasis on Etiquettes and Spiritual Value-oriented Guidance. In Taekwondo, etiquettes are viewed as the embodiment of the practicers' spirit. Each time before and after the practice and match, both parties must salute to each other. Through these etiquettes, practicers can develop good behaviors unconsciously. Besides that, popular Taekwondo also attaches great importance to the training of practicers' quality. By combining its own inner spirit and social morality, popular Taekwondo shows its value of existence and contribution to the society during the development.

Simple and Practical Technical System. Nowadays, people have so many pressures that they do not have enough time to take part in the complicate sports which have a lot of highly difficult movements and high level skills. With the characters of simple movements, short learning period, effective and practical, taekwondo is more easily accepted by people, especially teenagers. Moreover, its judgment criteria as well as training equipments are relatively simple and convenient. All these characters of Taekwondo meet the teenagers' psychological and physiological development needs, and arose their interest of exercising and offer them chance to involve in the sports personally.

Regular Taekwondo Sportswear and Rigorous Ranking System. In addition, the regular Taekwondo sportswear is another important factor in developing popular Taekwondo in China. As entering the training ground, practicers are required to wear the regular white Taekwondo sportswear which gives the impression of clean, capable, firm and tenacious. Although the style is simple, people find it is very attractive. Watching taekwondo's training, performance and competition, audience will be dazzled by its quick change of leg kicks and powerful strikes. All these visual impacts make audiences feel cheerful and eager to try, finally be fond of Taekwondo.

Promoting the Formation and Development of Personality. Most important function of sports is to influence people not only physiologically but also psychologically. Participating in sports activities needs spontaneity and patience for repeated practice, which are great influences on people's personality. For the participators, especially those teenagers, joining Taekwondo and making it an important part of their lives would be very helpful in enhancing physical fitness and skills. Furthermore, through Taekwondo competition, poomsae and capability test, practicer can train their qualities of toughness and perseverance. 


\section{Existing Problems of Popular Taekwondo in China}

After several decades of rapid development, popular Taekwondo has its solid mass base. According to incomplete statistics, there are over 3000 clubs and 5 million practicers. But some surveys on Taekwondo clubs and practicers show that there are still many problems in China's popular Taekwondo.

Uneven Distribution of Taekwondo Clubs. The distribution of Taekwondo clubs in China is very uneven, and there are relatively few large-scale ones. The main cause of this situation is the disequilibrium of Chinese regional economy development [6]. Nowadays, most of clubs are concentrated in economically developed areas, such as big cities, provincial capitals and developed city areas. It is very obvious that lacking of Taekwondo clubs in some cities and areas cannot meet the people's demand of practicing. And it has been becoming a big barrier in promotion of popular Taekwondo in China.

Imperfect Management System. Imperfect management system in Chinese Taekwondo clubs has aroused many practitioners' concern. The external problem in it is that there are multiple administrative departments whose duties are confusing and unclear, such as Education Bureau, Sports Bureau, and City Affairs Bureau and so on. And some of clubs are not independent legal entities with independent development space. Therefore, they just can submit to administrative interference rather than defend their proper interests legally when some problems occur. The internal problem is the shortage of executive and managerial personnel. This problem which is in forms of insufficient quantity, low academic qualification and inadequate experience of managerial personnel greatly obstructs the sustainable development efforts of popular Taekwondo in China.

Low Professional Quality of Coaches. Coaches' qualities are critical in many sports activities. Compared with those in foreign countries, Chinese primary coaches have fewer opportunities of learning and exchanging. As a result of that, teaching and training in most of clubs are poor, less standardized and scientific. Moreover, some coaches' used to major in other sports, such as Sanshou. Without constant learning of professional knowledge, they will definitely restrain and influence the development of popular Taekwondo.

Shortage of Competent Referees. To some degree, the rising and declining of a sport item are closely related to referees' capability. Compared to athletics Taekwondo, popular Taekwondo has less demand on referees, but it still needs them in poomsae performance and some friendly matches. As a young sports item, the referees' professional capability and ethics are relatively low. And the supervision and management system still has many flaws. These are greatly got in the way of development of popular Taekwondo.

Poor Condition of Taekwondo Clubs' Facilities. Taekwondo is a special sport item which needs sufficient space and high quality equipment and other conditions to be better carried out. But most of Chinese clubs' environment is not so good. With bad ventilation, shabby dressing room and substandard site, learners are unable to fully participate in learning, not to mention experience the culture atmosphere. Gradually, they will be bored and lose interest in taekwondo.

\section{Suggestive Measures for Development of Popular Taekwondo in China}

In order to better promote the development of Taekwondo, this paper puts forward some suggestive measures in view of the existing problems.

Building up High Standard Taekwondo Clubs through Multiple Financing Channels. With the improvement of people's living standard, there will be higher demand for the condition and equipment of Taekwondo clubs. In order to meet people's demand, building high standard 
Taekwondo clubs which can provide first-class services with good condition and equipment has become the major trend of development of popular Taekwondo in China [7]. Therefore, relevant government departments and Taekwondo associations should encourage, support and finance the development of key clubs, especially those in relatively less developed areas, which will be very helpful for the popularization and development of popular Taekwondo in China.

Standardizing and Strengthen the Administrative Management System. Nowadays, most of Taekwondo clubs are individual and private entities. In the market economy, as the survival and development are closely associated with economic profit, they will inevitably have some violations in competing for students and market share. Consequently, relevant administrative departments should standardize and strengthen management system, and eliminate the irregularities such as illegal operating and profiteering. Only in this way, popular Taekwondo can have a orderly and sound development.

Improving the Professional Quality of Taekwondo Coaches. Coaches' capability determines the quality of teaching and training. Popular Taekwondo clubs' coaches are lack of professional coach training and the valid certificate. They usually have some limitations in ideas, qualities, skills and training methods which have greatly hindered the training quality and effect [8]. So government sports departments and Taekwondo Association should strictly examine the qualification of coaches while providing appropriate trainings and organizing foreign exchanges. Taekwondo clubs should also be required to hold regular trainings and send their coaches to learn from other higher level coaching clubs. All in all, to improve the professional quality of Taekwondo coaches is an important aspect in its development which cannot be ignored.

Increasing Publicity Efforts of Popular Taekwondo. Strong media publicity is another necessary part in development of popular Taekwondo. Being an Olympic event, Taekwondo performance and competition can be well spread by using different media forms such as newspaper, magazine, book, radio and network. It also should strengthen the communication and cooperation with television stations, thus gain more recognition of the audience. In addition, we can display Taekwondo performance in evening parties and festival celebration to make more people understand its cultural connation and want to learn it.

\section{Conclusion}

Development of popular Taekwondo in China has more than 20 years, the establishment of Chinese Taekwondo Association and provincial association means that development of Chinese popular Taekwondo has entered into a new era. At the same time, it has played a important role in promoting and carrying forward the traditional East culture. Although there are still many problems need to be solved, with the effort and cooperation of practitioners, popular Taekwondo is bound to flourish in the near future in China.

\section{References}

[1] Kimun-youg: Taekwondo Textbook (Seoul-sung publishing co, Korea 2004).

[2] S.J. Hu and Z.H. Wang: Taekwond (Beijin Sport University Press, China 2009).

[3] J. Chen: Journal of Hangzhou Medical College. Vol.01 (2005) No.3. p.38.

[4] L.X. Chen, M.F. Zhang and L.H. Wu: Sports Sciences Researches. Vol.4(2000) No.1. p.37.

[5] Z.S. Ma, J.X. Su and G. Yong: Journal of Hebei Institution of Physical Education. Vol. 15(2001) No.3. p.60.

[6] M.H. Liu: Journal of Xi'an Physical Education University. Vol.23 (2006) No.2. p.37. 
[7] W.H. Liu: Sichuan Sports Science. Vol.4 (2010) No.4. p.14.

[8] W.H. Liu: Sichuan Sports Science. Vol.1 (2011) No.1. p.99. 